\title{
A Proposed Virtualization Technique to Enhance IT Services
}

\author{
${ }^{1}$ Dr Nashaat el-Khameesy \& ${ }^{2}$ Hossam Abdel Rahman Mohamed \\ ${ }^{1}$ Prof. and Head of Computers \& Information systems Chair, Sadat Academy \\ ${ }^{2}$ Computer \& Information System Dept - Sadat Academy, Computer \& Information System Dept - Sadat Academy for \\ management Science -Maady-Cairo-Egypt \\ ${ }^{1}$ Wessasalsol@gmail.com \& ${ }^{2}$ Hrahman@Transit.com.eg,HAbdel@Enr.gov.eg
}

\begin{abstract}
System virtualization is an antique art that will continue as long as applications need isolation and performance independence. Virtualization provides many benefits and greater efficiency in CPU utilization, greener IT with less power consumption, better management through central environmental control, more availability, reduced project timelines by eliminating hardware procurement, improved disaster recovery capability, more central control of the desktop, and improved outsourcing services. In this paper we will deliver new and enhanced IT services in less time and with greater agility. The goal of providing rapid near real-time response to customer requirements is in part being achieved by taking advantage of virtualization. Virtualization provides many benefits, including improved physical resource utilization, improved hardware efficiency, and reduced power and cooling expenses. However, it introduces another set of systems (virtual machines) that have to be controlled, provisioned, managed, updated, patched, and retired. This paper is organized into the following areas- An introduction to virtualization and its benefits, the Challenges of Virtualization techniques, an overview of the hardware virtualization technique, an overview of the software virtualization technique. The paper also proposes an effective and flexible distributed scheme with three phases, opposing to its predecessors. Our scheme achieves reducing the workload of a data center and continual service improvement.
\end{abstract}

Index Terms - Virtualization techniques, Virtual Machine, Hypervisors, Virtual Centre, Server Clustering

\section{Introduction}

Virtualization is, at its foundation, a technique for hiding the physical characteristics of computing resources from the way in which other systems, applications, or end users interact with those resources. This includes making a single physical resource (such as a server, an operating system, an application, or storage device) appear to function as multiple logical resources; or it can include making multiple physical resources (such as storage devices or servers) appear as a single logical resource. ${ }^{[1]}$ Virtualization is the approach of making one resource look like many or many resources look like one. For example, Virtual Machines have long been used to make a single computer look like more than one (and Virtual Storage was used to divide up the real storage in a computer for each of the virtual machines). Conversely, Grid Computing makes many independent computers work together as a single, virtual, computer. Networked storage (SAN and NAS technologies) likewise makes many separate units of storage appear as one large pool. And, in networking, Virtual LANs take a single Local Area Network and create logically-isolated sub-LANs (usually for performance or security purposes). [2] Virtualization provides compelling business value. Organizations can enhance IT service performance and scalability, achieving greater capacity (server MIPS, storage $\mathrm{GB}$, network $\mathrm{K} / \mathrm{M} / \mathrm{Gbps}$ ) than is available from a single instance of a resource. Efficiency is improved through consolidating the workloads of several underutilized resources (storage, servers, or networks) to improve resource utilization and reduce overall hardware, software, and management costs. Virtualization can also be used to enhance availability and security through containing untrusted applications (e.g. JAVA applets) in a controlled environment or isolating networks and servers into "zones" of trust. ${ }^{[3]}$ Virtualization can be viewed as part of an overall trend in enterprise IT that includes autonomic computing, a scenario in which the IT environment will be able to manage itself based on perceived activity, and utility computing, in which computer processing power is seen as a utility that clients can pay for only as needed. The usual goal of virtualization is to centralize administrative tasks while improving scalability and overall hardware-resource utilization. ${ }^{[4]}$

\section{Virtualization Techniques Challenges ${ }^{[5],[6],[7]}$}

While virtualization offers a number of significant business benefits, it also introduces some new management challenges that must be considered and planned for by companies considering a virtualization strategy. The key virtualization technique challenges for companies adopting virtualization include: 


\subsection{Bandwidth Implications}

Enterprises should make sure they have the appropriate network bandwidth for their virtualization requirements. For example, instead of one server using a $100 \mathrm{Mb}$ Ethernet cable, now 10 or even 100 virtual servers must share the same physical pipe. While less of a problem within the data center or for communication between virtual servers running in a single machine, network bandwidth is a significant issue for application streaming and remote desktop virtualization. These technologies deliver quite substantial traffic to end users, in most cases significantly higher than is required for standard-installed desktop computing. Streaming technologies, although in many cases more efficient than complete application delivery, also impose high bandwidth requirements.

\subsection{Image Proliferation}

Operating system and server virtualization can lead to a rapid proliferation of system images, because it is so much easier and faster to deploy a new virtual image than to deploy a new physical server, without approval or hardware procurement. This can impose very high management and maintenance costs, and potentially lead to significant licensing issues including higher costs and compliance risks. This proliferation also leads to significant storage issues, such as competing I/O and extreme fragmentation, requiring much faster and multichannel disk access, and more maintenance time, effort, and cost. Enterprises need to manage their virtual environment with the same level of discipline as their physical infrastructure, using discovery tools to detect and prevent new systems from being created without following proper process.

\subsection{Human Issues}

Enterprises should not underestimate the potential for human issues to affect their virtualization plans adversely. Virtualization requires a new set of skills and methodologies, not just within IT, but often (certainly in the case of application and desktop virtualization) in the end-user community. Perhaps most importantly, this new technology requires new and creative thinking, not just new training and skills.

\subsection{Policy-Based Management}

Enterprises should look to deploy automated policy based management alongside their virtualization strategy. Resource management, for example, should include automated policy-based tools for disk allocation and usage, I/O rates, CPU usage, memory allocation and usage, and network I/O. Management tools need to be able to throttle resources in shared environments, to maintain service levels and response times appropriate to each virtual environment. Administrators should be able to set maximum limits, and allocate resources across virtual environments proportionally. Allocations need to have the capability to change dynamically to respond to peaks and troughs in load characteristics. Management tools will also be required to automate physical to virtual, virtual to virtual, and virtual to physical migration.

\subsection{Security}

While virtualization can have many worthwhile security benefits, security also becomes more of a management issue in a virtualized environment. There will be more systems to secure, more points of entry, more holes to patch, and more interconnection points across virtual systems (where there is most likely no router or firewall), as well as across physical systems. Access to the host environment becomes more critical, as it will often allow access to multiple guest images and applications. Enterprises need to secure virtual images just as well as they secure physical systems. ${ }^{[8] \text {, }}$ [9].

\section{Virtualization Technique}

Virtualization refers to the abstraction of logical resources away from their underlying physical resources to improve agility and flexibility, reduce costs, and thus enhance business value. Virtualization allows a set of underutilized physical infrastructure components to be consolidated into a smaller number of better utilized devices, contributing to significant cost savings.

\subsection{Hardware Virtualization}

Hardware virtualization means that an application executes on virtualized hardware as opposed to physical hardware. "Virtual hardware" seems to be a contradictory term because the hardware is supposed to have a physical existence. Furthermore, the terms "virtual hardware" and "hardware virtualization" are used for different concepts in literature. We will discuss in this section the techniques of Memory virtualization, Power virtualization, Network virtualization and Storage virtualization.

\subsubsection{Network Virtualization Technique ${ }^{[1]]}$}

Network virtualization techniques can be leveraged in the networking infrastructure to achieve the same benefits obtained through server and storage virtualization. Moreover the network, especially in the data center, must also support this new dynamic environment where the computing and storage infrastructures are consolidated and virtualized, to meet new requirements (VM mobility, for example) and facilitate the delivery of IT services across heterogeneous network access technologies to multiple end user devices.

Network interface virtualization - these techniques refer to the Ethernet NICs and how they can be 
partitioned or aggregated. Sometimes these features are categorized as I/O Virtualization techniques.

Network Link virtualization - these techniques refer to how physical wires can be logically aggregated or partitioned to increase throughput and reliability or to provide traffic separation using the same physical infrastructure.

Network Node virtualization - these techniques refer to how network devices can be logically aggregated.

Data Centre Network-wide virtualization- these techniques extend the virtualization domain to the whole Data Centre Network and even to the WAN in case there are multiple Data Centers.

\subsubsection{Memory Virtualization Technique}

This involves sharing the physical system memory and dynamically allocating it to virtual machines. Virtual machine memory virtualization is very similar to the virtual memory support provided by modern operating systems. Applications see a contiguous address space that is not necessarily tied to the underlying physical memory in the system. The operating system keeps mappings of virtual page numbers to physical page numbers stored in the page tables. All modern x86 CPUs include a memory management unit (MMU) and a translation look-asidebuffer (TLB) to optimize virtual memory performance [12]

To run multiple virtual machines on a single system, another level of memory virtualization is required. In other words, one has to virtualize the MMU to support the guest OS. The guests OS continues to control the mapping of virtual addresses to the guest memory physical addresses, but the guest OS cannot have direct access to the actual machine memory. ${ }^{[13]}$ The VMM is responsible for mapping guest physical memory to the actual machine memory, and it uses shadow page tables to accelerate the mappings. As depicted by the red line in Figure 1.

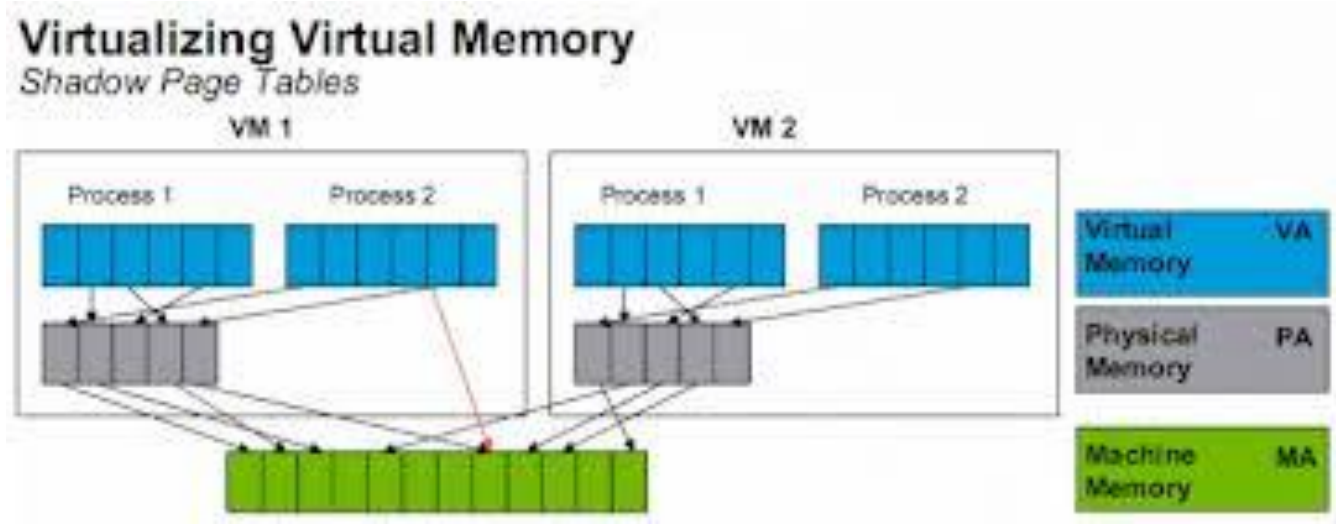

Fig. 1: VMM uses TLB hardware to map the virtual memory directly to the machine memory

The VMM uses TLB hardware to map the virtual memory directly to the machine memory to avoid the two levels of translation on every access. When the guest OS changes the virtual memory to physical memory mapping, the VMM updates the shadow page tables to enable a direct lookup. MMU virtualization creates some overhead for all virtualization approaches, but this is the area where second generation hardware assisted virtualization will offer efficiency gains. ${ }^{[14] \text {. }}$

\subsubsection{Power Virtualization Technique}

The Power VM platform is the family of technologies, capabilities, and offerings that deliver leading virtualization on the Power Systems .It is the new umbrella branding term for Power Systems Virtualization (Logical Partitioning, MicroPartitioning ${ }^{\mathrm{TM}}$, Power Hypervisor, Virtual I/O Server, Live Partition Mobility, Workload Partitions, and so on) [15], [16] as with Advanced Power Virtualization in the past, Power VM is a combination of hardware enablement and value-added software. There are three versions of Power VM, suited for different purposes:

Power VM Express Edition- This edition is intended for evaluations, pilots, proof of concepts, generally in single-server projects.

Power VM Standard Edition- This edition is intended for production deployments, and server consolidation.

Power VM Enterprise Edition- the Enterprise Edition is suitable for large server deployments such as multiserver deployments and cloud infrastructure.

\subsubsection{Storage Virtualization Technique}

Storage virtualization refers to the process of completely abstracting logical storage from physical storage. The physical storage resources are aggregated into storage pools, from which the logical storage is created. It presents to the user a logical space for data storage and transparently handles the process of mapping it to the actual physical location. This is currently implemented inside each modern disk array, 
using the vendor's proprietary solution. However, the goal is to virtualize multiple disk arrays, made by different vendors, scattered over the network, into a single monolithic storage device, which can be managed uniformly.

Currently, storage is virtualized by partitioning the SAN into separate partitions called Logical Unit Numbers (LUNs). Each LUN can only be connected to one server at a time. ${ }^{[17]}$ This SAN is connected to the server through a Fibre Channel connection switch.

The advantages of SAN virtualization are:(Centralized stored data, Ease of backup of stored data, Ability to remap a LUN to a different server in case of server failure, Storage appears local to servers and to users, Improves utilization and reduces storage growth, Reduces power and cooling requirements)

\subsection{Software Virtualization}

Software virtualization is the virtualization of applications or computer programs. The concept is similar to hardware virtualization where physical machines are simulated as virtual machines. Software virtualization involves creating a virtual layer or virtual hard drive space where applications can be installed. From this virtual space, applications can then run as though they have been installed onto host OS. We will discuss in this section the techniques Application virtualization, Operating System virtualization, Desktop virtualization and Server virtualization.

\subsubsection{Application Virtualization Technique}

An Application Virtualization transforms applications into virtualized, network-available services resulting in dynamic delivery of software that is not installed, minimizes application conflicts, and reduces costly application compatibility testing. Users and their application environments are no longer machinespecific, and the machines themselves are no longer user-specific, enabling IT to be flexible and responsive to business needs, and significantly reducing the cost of
PC management, including application and operating system (OS) migrations ${ }^{[18] \text {. }}$

\section{Application Virtualization Advantages:}

Minimize application conflicts and regression testingBy reducing the requirement to install applications on desktops or terminal servers, and shielding the OS and applications from changes created when applications are installed, Application Virtualization minimizes the problems that hinder application deployments. This also reduces lengthy application to application regression testing. Now, applications that would traditionally conflict with each other can easily co-exist on a single desktop or Terminal Services session.

Simplify OS migrations and patching- Turn timeconsuming, tedious migration and patching projects into largely automated, conflict-free processes.

Build business continuity for applications- Replicate our virtualized applications like any other enterprise data to maintain a rapid failover plan for your applications, significantly cutting end-user downtime. If you configure Application Virtualization user profiles to persist on the network, all user-specific virtual application preferences can also easily be replicated to a backup site.

\subsubsection{Server Virtualization Technique}

Server virtualization is a method of abstracting the operating system from the hardware platform. This allows multiple operating systems or multiple instances of the same operating system to coexist on one or more CPUs. A hypervisor or virtual machine monitor (VMM) is inserted between the operating system and the hardware to achieve this separation. These operating systems are called "guests" or "guest OSs." The hypervisor provides hardware emulation to the guest operating systems. It also manages allocation of hardware resources between operating systems. [19], [20], [21]

Currently there are three common types of hypervisors: type 1, type 2, As we see Figure2

\section{depicts the three hypervisor types.}

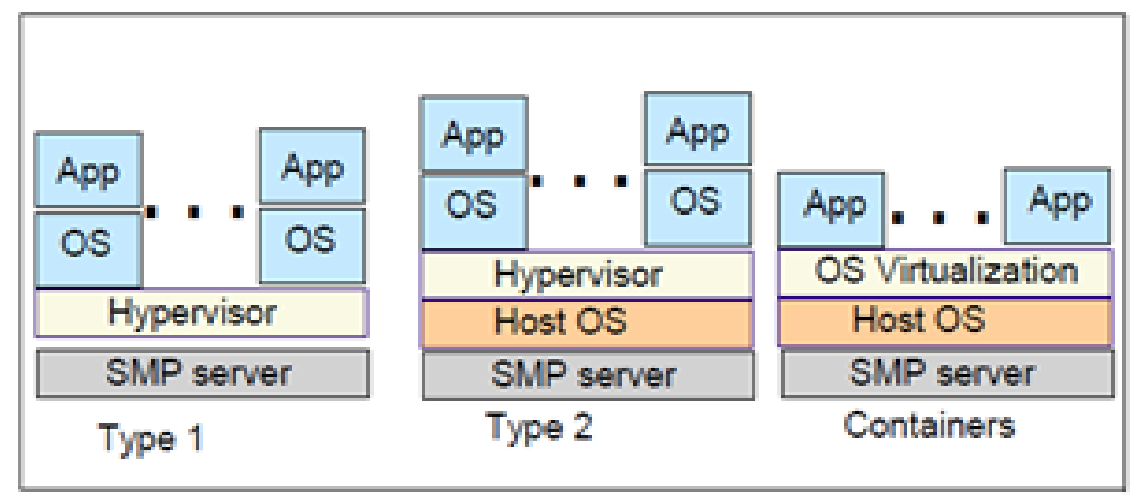

Fig. 2: The types of hypervisors 
Type 1 - Virtualization code that runs directly on the system hardware that creates fully emulated instances of the hardware on which it is executed. Also known as "full," "native," or "bare metal."

Type 2 - Virtualization code that runs as an application within a traditional operating system environment that creates fully emulated instances of the hardware made available to it by the traditional operating system on which it is executed. These are also known as "hosted" hypervisors.

Containers - Virtualization code that runs as an application within a traditional operating system that creates encapsulated, isolated virtual instances that are pointers to the underlying host operating system on which it is executed. This is also known as "operating system virtualization."

\subsubsection{Desktop Virtualization Technique ${ }^{[22]}$}

Desktop Virtualization enhances deployment and management of Virtual PC images on a Windows Desktop while also providing a seamless user experience on a Virtual PC environment independent of the local desktop configuration and operating system.

\section{Desktop Virtualization Advantages}

1) Easily deploy managed Virtual PCs to any desktop, even in less controlled environments such as subsidiaries, branch offices and offshore operations.

2) Drive business continuity by rapid reconstitution of corporate Desktops.

3) Accelerate migration to the Windows by minimizing application- to-OS compatibility issues.

4) Speed user adoption of desktop virtualization by making Virtual PCs "invisible" to users.

5) Reduce IT investment in desktop image management by delivering virtual images independent of hardware or the local desktop configuration.

\section{Design Goals}

To Decrease the workload for data center and ensure continual service improvement, we aim to design efficient mechanisms for reducing the workload of a data center and business Continuity verification and achieve the following goals:

\subsection{On-Demand Data Center Workload Streaming}

By Delivers the OS, applications, and server configuration information in a real-time stream, maximizing performance and minimizing network load,
Risk - free server workload rollout, Maximizes boot performance, Minimizes network load, Provisions multiple servers from a single virtual disk image, Minimizes storage and image management requirements, Ensures server build consistency, Allows switching of server workloads in the time it takes to reboot, Maximizes flexibility by enabling real-time changes to data centre workloads, Reduces the number of servers needed to support disaster recovery \& business continuity requirements.

\subsection{Improved Total Cost of Ownership}

By decreasing management costs and increasing asset utilization, we can experience a rapid return on investment (ROI) with virtualization. In addition, by virtualizing resources and making them easier to migrate or fail over to other physical devices or locations, you can enhance system availability and help lower the cost and complexity of disaster-recovery solutions.

\subsection{Increased Flexibility}

Virtualization supports the pooling of resources that can be managed centrally through an enterprise hub to better support changing business requirements dynamically.

\subsection{Enabled Access through Shared Infrastructure}

By providing a resilient foundation and shared infrastructure that enables better access to infrastructure and information in support of business applications and service-oriented architectures (SOA).

\subsection{Energy Efficiency}

We can help control infrastructure sprawl through the deployment of virtual servers and storage that run securely across a shared hardware environment. Virtualization not only helps with server consolidation, but also server containment when deploying new systems. Consolidating to a virtual infrastructure can enable you to increase server utilization rates from $5 \%$ to $15 \%$ to over $70 \%$, thus helping improve ROI. In addition, a simplified infrastructure can help lower manage costs with a common management platform and tooling.

\subsection{Rapid Application Deployment}

We can use the virtualization can help enable rapid infrastructure provisioning (ex. Minutes compared to days). It can help developers to speed application test and deployment, enhance collaboration and improve access to the infrastructure. The ease and flexibility of creating and reconfiguring guest operating systems means that development and test environments can realize significant benefits from virtualization. 


\subsection{Improved Disaster Recovery}

Virtualization can help IT Dept secure and isolate application workloads and data within virtual servers and storage devices for easier replication and restoration. This added resiliency can provide IT managers with greater flexibility to maintain a highly available infrastructure while performing planned maintenance, and to configure low-cost disaster-recovery solutions.

\subsection{Managing a Virtualized Infrastructure}

We can address configuration, deployment, monitoring, workload management and additional management function in a consistent and common way across their infrastructures. This can help simplify problem determination, increase productivity and lower management costs.

\section{Proposed Solutions}

The Virtualization can reduce system and workload of a data center. Further, virtualization can be implemented in phases allowing for capital expense spending flexibility. The purpose of virtualization is to increase efficiency and reduce costs, with each phase addressing different aspects of specific resources. Phase I should include hardware consolidation and virtualization of the web and application tiers along with the commoditization of the database tier. The specific focus is on reducing hardware maintenance costs, (End of Support Life) hardware and reducing power consumption. The main objective is to increase efficient use of CPU capacity and to create system flexibility and resiliency. Phase II should include implementation of desktop virtualization with a primary focus on change management issues and reducing the impact of human error. Further, an emphasis on training, organizational restructuring and group consolidation to support operational technologies implemented in Phase 1 should be incorporated. Phase III should include full data tier virtualization and disaster recovery focusing on full multi-site recovery in a cost effective manner.

Table: 1 Proposed Solution Phases

\begin{tabular}{|c|c|c|}
\hline & Target Resource and Objective & Focus \\
\hline Phase I & $\begin{array}{l}\text { Network Infrastructure } \\
\text { Servers/Web/Application virtualization }\end{array}$ & $\begin{array}{l}\text { Reduction in hardware and maintenance costs } \\
\text { Resolve issues } \\
\text { Improve system flexibility, resiliency and efficiency in power consumption and CPU } \\
\text { capacity }\end{array}$ \\
\hline Phase II & $\begin{array}{l}\text { Human Capital } \\
\text { Desktop virtualization and Human } \\
\text { Capital Investment }\end{array}$ & $\begin{array}{l}\text { Improve Efficiency in Time Management } \\
\text { Train/Retrain labor in new technology to reduce knowledge gap } \\
\text { Organizational restructuring to reduce labor costs }\end{array}$ \\
\hline Phase III & $\begin{array}{l}\text { Multi-site Infrastructure } \\
\text { Disaster Recovery }\end{array}$ & $\begin{array}{l}\text { Fully capitalize on virtualization through integrating sites. } \\
\text { Reduce hardware and maintenance } \\
\text { Costs at disaster recovery site } \\
\text { Eliminate EOSL issues at Disaster } \\
\text { Recovery site } \\
\text { Enhance disaster recovery by reducing down time and implementing full recovery }\end{array}$ \\
\hline
\end{tabular}

\subsection{Planning Phase I:}

The solution we offered in this paper was effectual to choose the more complex option of changing the IP addresses of the servers. The alternative was to assume VLAN was stretched or moved during failover which would be simple to document but we felt more value would be derived from recoding the IP address of each server.

From a high level perspective we selected a discrete IP address range in each site for both the VMware Infrastructure management infrastructure and the guest operating systems. I.e. in a failover we change all of the protected virtual machines IP address.

From a VMware Infrastructure perspective all of the backing infrastructure is set up as part of the build out and remains static during the failover events so this aspect is very straightforward.
At each site we separated the virtual machine and Management Infrastructure onto two separate subnets/VLANS and provided a third for the router to use for inter site traffic.

\subsubsection{Network infrastructure:}

Modern applications are almost always dependent on networking to some degree or another and so physically moving a service from one site to another represents a networking challenge whether the services are hosted in virtual machines or physical machines alike. Network addressing is not a general location independent mainly due to poor application use of network addressing technologies that have been available for many years. However we have to deal with applications and configurations which depend on hard coded IP addresses. 
The solution we offered in this paper was effectual to choose the more complex option of changing the IP addresses of the servers. The alternative was to assume VLAN was stretched or moved during failover which would be simple to document but we felt more value would be derived from recoding the IP address of each server.

- From a high level perspective we selected a discrete IP address range in each site for both the VMware Infrastructure management infrastructure and the guest operating systems. I.e. in a failover we change all of the protected virtual machines IP address.

- $\quad$ From a VMware Infrastructure perspective all of the backing infrastructure is set up as part of the build out and remains static during the failover events so this aspect is very straightforward.

- At each site we separated the virtual machine and Management Infrastructure onto two separate subnets/VLANS and provided a third for the router to use for inter site traffic.

\subsubsection{Infrastructure Services}

Demand for virtualization of networks, servers, storage, desktops and applications continue to increase. Cisco is assuming the virtualization of DC services is now the default building block of the data center, and has organized its portfolio to hasten this transition.

- To ease information overload a cohesive optimization system and process was chosen. For this proposed study, using Citrix Xen Application and Oracle RAC Clusters on the Cisco Unified Computing System with Citrix Xen Server VMware enterprise software works to address two goals. First, this system eases the real time issue of a knowledge base shift through a cohesive and simplified deployment ${ }^{[23]}$. Second, high performance is cost effective, reducing budget concerns.

- Efficiency and performance through the Cisco UCS is also realized through the Cisco partnership with Oracle RAC. Oracle has a "mission critical" focus in their database and application technologies ${ }^{[24]}$ which are highly relevant to large corporations as well as the university. The Cisco UCS memory capability coupled with a scalable storage system opens the performance floodgates to Oracle database environments allowing for unheard of work and performance loads. Previous workload performance testing " included a realistic mix of OLTP and DSS workloads, which generated a sustained load on the eight-node Oracle RAC configuration for a period of 72 hours... far exceeding the demands of typical database deployments" . The results showed that processors were barely at 50 percent capacity and port utilization was at 40 percent. The expansion potential of the system allows the organization a cost effective way to scale up, if (or when) the need arises.

- Database administrators are now relieved of the time consuming task of configuring each hardware element in the stack independently by virtue of the Cisco UCS Manager. Further, the UCS Manager consistently and accurately configures servers through service-based profiles thereby eliminating human error almost completely and simplifying the scaling process. Finally, the "wire once" configuration simplifies cabling in the rack as network cabling is done once with changes implemented through software, reducing the potential error rate in the cabling process. Reduction in human error alone allows for better-managed and more effective work time resulting in higher labor efficiency ${ }^{[25]}$

\subsection{Planning Phase II:}

Phase two of optimization can be a springboard to catapult newer employees into new technology and specialized positions all but eliminating seniority and property rights to specific positions. The notion being that new technology creates new positions with specialized skills and requirements for the job. The motivation for current employees will be to embrace training, technology and apply for positions based on knowledge and merit (not politics) or risk lay-off.

Technically, this is the least difficult phase as the focus is on desktop virtualization. However, this can be the most difficult phase for managing. Virtualization reduces desktop replacement costs, power consumption and costs related to desktop support. However, desktop virtualization equates to labor reduction and salary savings.

\subsubsection{Desktop Virtualization}

The second half of phase two is the actual implementation of desktop virtualization. The end result being reduced complexity and risk from remote access, reduced power consumption, and reduction in endpoint risk. A reduction in effort and risk from patching and reducing sensitive data protection efforts and risks should also be evident. This is realized through leveraging knowledge from the consolidation and virtualization put in place during phase I. With desktop virtualization, power consumption is assumed to drop $75 \%$ based on data and test results taken from phase I. Reduced effort and risk as well as increased host security is assumed since there is no longer a requirement for the end user to interact with their local host to assure patching. Further, due to a reduction in the data footprint, there is a reasonable assumption of 
increased data security, as the data no longer resides at the end point desktop or laptop. ${ }^{[26]}$

\subsection{Planning Phase III:}

In the best of all possible worlds, organizations would implement backup and recovery processes for all server workloads regardless of their perceived criticality. Moreover, these recovery processes would be fast, have minimal impact on production operations and would be recoverable with a high level of data integrity. In reality, disaster recovery needs must always be weighed against the fiscal need for cost-effectiveness. ${ }^{\text {[27] }}$

To meet a number of the desired design criteria, we will use a two-site approach with an active Virtual Centre instance at each location. These are referred to as Site 1 and Site 2 throughout the documentation. Both sites are setup to have an active workload required to run the day-to-day activities present at each site. Additionally, at Site 1 there is a group of services that must be capable of being failed over to Site 2 and run there successfully. These are known as the protected services. The design will also accommodate work loads from Site 2 hosted on Site 1 although this was not implemented at this stage.

As shown in figure 3, the logical view of the configuration we have used in this paper. Site 1 holds virtual machines from an imaginary HR and Finance department and Site 2 hosts a number of Developer and other production services. Virtual machines can be executed in either site with protection for the machines in Site 1.

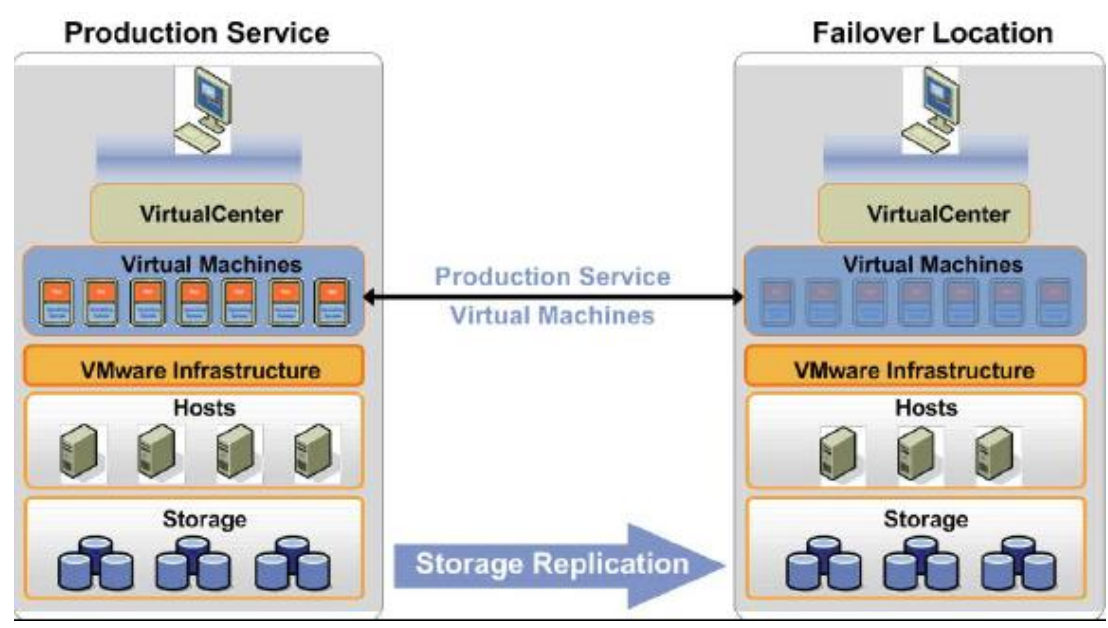

Figure 3 Site 1 holds virtual machines and Site 2 hosts a number of Developer and other production services

\section{Virtual Centre Management Server Architecture}

Virtual Centre Management Server provides a convenient centralized management cockpit for the data centre. It aggregates physical resources from multiple Server installations and presents a central collection of simple and flexible resources for the system administrator when provisioning virtual machines in the virtual environment.

Core Services are basic management services for a virtual data center. They include services such as:

- VM Provisioning - Guides and automates the provisioning of virtual machines

- Host and VM Configuration - Allows the configuration of hosts and virtual machines

- Resource and Virtual Machine Inventory Management - Organizes virtual machines and resources in the virtual environment and facilities their management

- Statistics and Logging - Logs and reports on the performance and resource utilization statistics of data center elements, such as virtual machines, hosts and clusters

- Alarms and Event Management - Tracks and warns users on potential resource over-utilization or event conditions

- Task Scheduler - Schedules actions such as VMotion to happen at a given time.

\section{Reliability of Virtualization and Full Tolerance}

Reliability - Virtualization is very reliable. Virtualization can help us eliminate planned downtime, ensure data protection, deliver high availability and be better prepared for disaster recovery. The FaultTolerance (FT) feature of VMWare, helps protect against physical hardware failures, including network card failures, storage path failures and other hardware failures.

Full tolerance- During server consolidation efforts, one of the key concerns often is the impact failure of a virtualization host might have to data centre operations. 
High availability ensures critical VMs are automatically restarted on another physical host should the original host running the VM unexpectedly fail. This can reduce the amount of downtime for the workload, as well as eliminate the need for administrative intervention. ${ }^{[28]}$ XenServer has high-availability features that offer granular policies governing the behavior of specific VMs after a host failure. Additionally, both Citrix and VMware offer options for fault tolerance, a feature that maintains mirrored instances of VMs running on separate hosts. In the event of a host failure, the mirrored instance can maintain continuity of the workload. Citrix has partnered with several third-party vendors, such as Marathon Technologies and Stratus, to create more robust fault tolerance options for XenServer.

\section{Conclusions}

This paper proposes a Virtualization Techniques Model for reducing the workload of a data center and reducing complexity and costs while improving system capacity, flexibility, and efficiency. In addition, the proposed model improves service Continuity. [29], [30], [31], [32]

We have proposed an effective and flexible virtualization solutions Infrastructure that is architected from the ground up to enable organizations to bring the benefits of virtualization to the entire enterprise platform, including server, storage and networking systems. The virtualization solutions Infrastructure not only enables organizations to build an entire virtual data centre that matches the capabilities available in the physical world, but it goes one step further by offering the flexibility and control that is physically costprohibitive or impossible.

- We proposed an Implemented plan which is easy to manage and scalable for future growth, resulting in reduced administrative burden.

- Our proposed An easier, faster and consistent centralized server deployment and protection of Virtual Machines

- We have discussed how System Virtualization Networking can help resolve network configuration challenges introduced by server virtualization.

- We have discussed management capabilities for virtualization Techniques

- We have discussed how virtualization can help automate network configuration in virtualized environments, and how it enhances network control by making the network aware of virtual machines.

- With a whole suite of complementary virtualization and management services such as virtual VMware VMotion, VMware DRS, VMware HA and VMware Consolidated Backup,
VMware Infrastructure is the only offering that delivers a complete solution rather than piecemeal approach for customers to build a data center in a virtual environment.

\section{References}

[1] Susanta Nanda Tzi-cker Chiueh and Stony Brook, A Survey on Virtualization Technologies. RPE Report, pages 1-42, 2005

[2] Amit singh, "An introduction to virtualization”,http://www.kernelthread.com/public ations/virtualization, 2004.

[3] Mueen Uddin,Azizah Abdul Rahman,Virtualization Implementation Model for Cost Effective \& Efficient Data Centers, (IJACSA) International Journal of Advanced Computer Science and Applications, Vol. 2, No.1, January 2011

[4] T. Daim, J. Justice, M. Krampits, M. Letts, G. Subramanian, M. Thirumalai, "Data center metrics An energy efficiency model for information technologu managers", Management of Environmental Quality, Vol.20 No.6, 2009.

[5] Chalapathi Valupula,R. Lakshman Naik,Sunitha Muppa, Scheduling in Virtual Infrastructure for Highthroughput Computing ,International Journal of Computer Science, Engineering and Applications (IJCSEA) Vol.2, No.3, June 2012

[6] Virtualization: State of the Art Version 1.0, April 3, 2008 Copyright @ 2008 SCOPE Alliance.

[7] VMWare. Transparent Previrtualization info page. http://www.vmware.com/interfaces/paravirtualizati on.html.

[8] Haletky, Edward. (2009). VMware vSphere ${ }^{\mathrm{TM}}$ and Virtual Infrastructure Security: Securing the Virtual Environment. Prentice Hall. Retrieved June 12, 2010, from http://www.safaribooksonline.com

[9] Flavio Lombardia, Roberto Di Pietrob, Secure virtualization for cloud computing Journal of Network and Computer Applications Volume 34, Issue 4, July 2011, Pages 1113-1122

[10] Gai, Silvano; Salli, Tommi and Anderson, Roger (2010). Cisco Unified Computing System (UCS) (Data Center): A Complete Reference Guide to the Cisco Data Center Virtualization Server Architecture. Indianapolis, IN: Pearson Education, Cisco Press. Retrieved June 7, 2010, from http://www.safaribooksonline.com

[11] P. Viana. Exploring Memory Hierarchy with ArchC. Symposium on Computer Architecture and High Performance Computing, pages 2-9, 2003.

[12] C. Arajo. Platform Designer: An Approach for Modeling Multiprocessor Platforms based on 
SystemC. Journal of Design Automation for Embedded Systems, 10(4):253-283, Springer 2003.

[13] G. Neiger. Intel Virtualization Technology: Hardware Support for Efficient Processor Virtualization. Intel Computer Journal, 10:166178, August 2006.

[14] K. Adams and O. Agesen, "A comparison of software and hardware techniques for $\mathrm{x} 86$ virtualization" in ASPLOS-XII: Proceedings of the 12th international conference on Architectural support for programming languages and operating systems (San Jose, California, USA, 2006), ACM, pp. 2-13.

[15] NEIGER, G., SANTONI, A., LEUNG, F., RODGERS, D., AND UHLIG, R. Intel virtualization technology: Hardware support for efficient processor virtualization. Intel Technology Journal 10, 3 (2006).

[16] Adrian De Luca, Mandar Bhide, Storage Virtualization For Dummies, Wiley Publishing, Inc. 111 River Street Hoboken, NJ 07030-5774 Copyright (C) 2010 by Wiley Publishing, Inc., Indianapolis, Indiana

[17] He, Yu,Hu,Yuhong\&Wang, Yibo. (2007). Application of virtualization technology in data center of campus network. Journal of University of Electronic Science and Technology of China. 2007,36(6):1461-1464.

[18] James E. Smith and Ravi Nair. The Architecture of Virtual Machines. IEEE Computer, 38(5):32-38, 2005.

[19] Y. Ha, S. Vernalde, P. Schaumont, M. Engels, R. Lauwereins, and H. De Man, "Building a Virtual Framework for Networked Reconfigurable Hardware and Software Objects," Journal of Supercomputing, vol. 21, no. 2, pp. 131-144, February 2002.

[20] Wang,Jianhong. (2008). The analysis on computer virtualization of linux. Journal of Hubei Normal University (Natural Science).2008, 28(1): 62-64.

[21] A. Whitaker, M. Shaw, and S. D. Gribble. Denali: Lightweight Virtual Machines for Distributed and Networked Applications. Technical Report 02-0201, University of Washington, 2002.

[22] K. A. Fraser, S. M. Hand, T. L. Harris, I. M. Leslie, and I. A. Pratt. The Xenoserver computing infrastructure. Technical Report UCAM-CL-TR552, University of Cambridge, Computer Laboratory, Jan. 2003.

[23] MENON, A., SANTOS, J. R., TURNER, Y., JANAKIRAMAN, G., AND ZWAENEPOEL, W. Diagnosing performance overheads in the Xen virtual machine environment. In First ACM/USENIX Conference on Virtual Execution Environments (VEE’05) (June 2005).
[24] Cisco. (2010). Deploying Oracle Real Application Clusters on the Cisco Unified Computing System with EMC CLARiiON Storage. White Paper. Cisco.com. Retrieved September 1, 2010, http://www.cisco.com/en/US/prod/collateral/ps102 65/ps10280/white_paper_c11- 562881.pdf.

[25] MENON, A., COX, A. L., AND ZWAENEPOEL, W. Optimizing network virtualization in Xen. In USENIX Annual Technical Conference (June 2006).

[26] LEVASSEUR, J., UHLIG, V., CHAPMAN, M., CHUBB, P., LESLIE, B., AND HEISER, G. Previrtualization: Slashing the cost of virtualization. Technical Report 2005-30, Fakult"at f "ur Informatik, Universit”at Karlsruhe (TH), Nov. 2005.

[27] VMware ${ }^{\circledR}$ Fault Tolerance Recommendations and Considerations on VMware vSphere ${ }^{\mathrm{TM}} 4$ http://fault_tolerance_recommendations_considera tions_on_vmw_vsphere4.pdf

[28] http://www.ibm.com/support/techdocs/atsmastr.nsf /WebIndex/WP101520

[29] http://www.ibm.com/support/techdocs/atsmastr.nsf /WebIndex/WP101597

[30] Enabling a Dynamic Datacenter with Microsoft Virtualization , Published: June 2008, http://download.microsoft.com/download/a/7/a/a7a d1735-40d7-4508-9462-

bcb79577b17b/virtualization_whitepaper_v2_1.pdf

[31] Citrix- Advanced virtualization management for Microsoft virtualization, http://www.networkworld.com/chatterbox/citrix/di mension3/Citrix_Essentials_for_HyperV_Product_Overview_March_2009.pdf

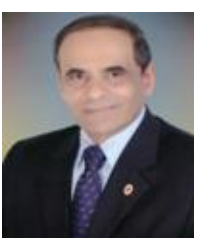

Dr Nashaat el-Khameesy: Prof. and Head of Computers \& Information systems Chair, Sadat Academy

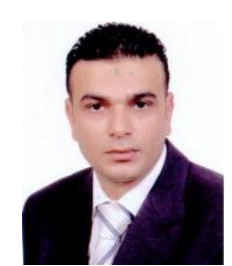

Hossam Abdel Rahman Mohamed: Post-graduate student for degree of Master of computer science \& information system - Sadat Academy Dept Computer and Information System,Maady , Cairo , Egypt - He is currently position Data Center Operation Team leader at Trans IT Company - Ministry of Transportation 